\title{
.
}

\section{Non-equilibrium conditions inside rock pores drive fission, maintenance and selection of coacervate protocells}

\author{
Alan laneselli, ${ }^{1,2}$, Damla Tetiker', Julian Stein ${ }^{1,2}$, Alexandra Kühnlein ${ }^{1,2}$, Christof B. Mast $\oplus^{1,2}$, \\ Dieter Braun ${ }^{1,2} \otimes$ and T.-Y. Dora Tang ${ }^{3}{ }^{3 凶}$
}

Key requirements for the first cells on Earth include the ability to compartmentalize and evolve. Compartmentalization spatially localizes biomolecules from a dilute pool and an evolving cell, which, as it grows and divides, permits mixing and propagation of information to daughter cells. Complex coacervate microdroplets are excellent candidates as primordial cells with the ability to partition and concentrate molecules into their core and support primitive and complex biochemical reactions. However, the evolution of coacervate protocells by fusion, growth and fission has not yet been demonstrated. In this work, a primordial environment initiated the evolution of coacervate-based protocells. Gas bubbles inside heated rock pores perturb the coacervate protocell distribution and drive the growth, fusion, division and selection of coacervate microdroplets. Our findings provide a compelling scenario for the evolution of membrane-free coacervate microdroplets on the early Earth, induced by common gas bubbles within heated rock pores.

C ompartmentalization is a key feature of modern biological systems and has been hypothesized to play an important role during the origin of life by spatially localizing molecules and facilitating the first chemical reactions ${ }^{1,2}$. One viable route to compartmentalization is via liquid-liquid phase separation of oppositely charged polyelectrolytes in aqueous solution ${ }^{3}$. This process leads to the formation of membrane-free chemically enriched droplets. These coacervate microdroplets are intriguing protocell models as they form with little chemical identity under a broad range of physico-chemical conditions ${ }^{4}$; they localize and concentrate a range of different molecules ${ }^{5-7}$ and exhibit molecular selectivity by partitioning ${ }^{8-10}$. In addition, coacervate droplets facilitate the assembly of fatty acid bilayers on their outer surface ${ }^{11}$ and readily support catalytic reactions such as primitive RNA catalysis ${ }^{12-14}$. This provides a pathway to membrane-bound compartmentalization as observed in modern biology and a connection to the RNA-peptide world hypothesis.

Fusion events, division and maintenance of coacervate protocells would have been essential for the evolution of compartmentalized molecules. Fusion and growth of protocells are necessary for the exchange of molecules and genetic material ${ }^{15}$ and it has been shown that the incorporation of free components by direct fusion with other protocells ${ }^{16}$ or by external electric fields ${ }^{17}$ can be achieved in a laboratory setting. In solution, these coacervate droplets will tend to coalescence, eventually forming a coacervate bulk macrophase $\mathrm{e}^{18,19}$, which limits their role as protocells. The division of coacervate protocells is required to transfer molecular information to succeeding daughter protocells that can pass evolutionary advantages to the next generation. To achieve division, modern cells make use of a complex machinery of regulatory proteins, scaffold proteins, enzymes and chemical messengers ${ }^{20}$. In the prebiotic world, division must have relied on other factors. Some studies suggest that division of lipid-based vesicles can be triggered by osmotic changes $^{21}$, chemical changes ${ }^{22}$, temperature ${ }^{23}$ and shearing forces ${ }^{24}$. By comparison, less is known about the division mechanisms of membrane-free coacervate-based protocells that are chemically enriched. One theoretical study predicts that budding of chemically active membrane-free droplets is achieved by the flux of substrate and product across the interface which lies in a particular surface-tension regime ${ }^{25}$. Despite this prediction, there has been no experimental realization of fission of membrane-free protocells with or without chemical input. Furthermore, it has still not been experimentally shown how they would behave under prebiotically plausible non-equilibrium conditions.

To this end, pores in a thermal gradient provide a unique, facile and prebiotically feasible route to perturbing the system away from its equilibria ${ }^{26}$. Here, capillary flows induced by heat fluxes within millimetre-sized pores have been shown to accumulate molecules based on their size at the gas-water interface of gas inclusions. Simulations and experiments show that there are two main forces acting at the interface: capillary flows from the cold to the warm side and perturbative fluxes after the precipitation of water ${ }^{27,28}$. These forces induce rapid movements of particles, driving their contact and fusion. Under these conditions, lipid molecules accumulate at the interface to create vesicular structures and undergo fission driven by Marangoni flows and convection. These previous studies indicate that the growth, division and maintenance of coacervate droplets could be manipulated by the physical flows within thermal pores.

In this Article, we study the effect of out-of-equilibrium conditions provided by heated pores containing gas bubbles, a common primordial scenario ${ }^{26}$, on the growth and division mechanisms of complex coacervate microdroplets formed by mixing polyanionic (carboxymethyl dextran (CM-Dex), adenosine 5' -triphosphate (ATP)) and polycationic (polydiallyldimethylammonium chloride (PDDA), poly-L-lysine (pLys)) species. Even though the coacervates 
in this study might not be generated from prebiotically relevant molecules, they provide a robust model system for reconciling the general role of heat-induced out-of-equilibrium systems on coacervate microdroplets.

We show that the accumulation of coacervate-forming components at the gas-water interface of the gas bubble ${ }^{28}$ drives growth by fusion of the coacervate microdroplets. Droplets of up to $300 \mu \mathrm{m}$ in size are formed and maintained over time. This property is not observed under equilibrium conditions where droplets coalesce to eventually form a single coacervate macrophase (Supplementary Fig. 5.1 $)^{18,19}$. Intriguingly, the microfluidic water cycle induced by the thermal gradient ${ }^{27}$ creates perturbative fluxes at the gas-water interface that lead to the fission and fragmentation of the coacervate droplets using purely physical processes (Fig. 1a-c). This offers direct evidence that physical forces within a confined environment are sufficient to provide the mechanism of membrane-free protocell division without complex machinery or targeted chemical reactions. Furthermore, the environment provided the ability to create and select for separate populations of droplets with different chemical composition. Specifically, the out-of-equilibrium conditions were able to overcome the intrinsic preference of RNA to coacervate with pLys (ref. ${ }^{29}$ ), yielding RNA:pLys droplets also enriched with CM-Dex at the gas-water interface. In the bulk, the coacervate droplets were formed mainly by RNA and pLys. This means that the thermal gradient, in combination with the gas bubble, led to the creation and spatial segregation of two different populations of coacervate droplets with different composition: oligonucleotide:polypeptide (RNA:pLys) coacervate droplets in the bulk and sugar:oligonucleotide:polypeptide (CM-Dex:RNA:pLys) droplets at the gas-water interface.

We present the proposed mechanisms as a prebiotic model for membrane-free protocell growth, division and evolution, since the only requirements are simple and ubiquitous physical conditions that could be found inside heated rock pores on the early Earth.

\section{Results}

The gas-water interface accumulates coacervate droplets and facilitates fusion. To characterize the effect of non-equilibrium perturbations on coacervate microdroplets, we experimentally recreated a heated rock pore filled with liquid and gas bubbles as described previously ${ }^{27,28}$. In brief, a polytetrafluoroethylene (PTFE) sheet $(250 \mu \mathrm{m}$ thick) cut with sharp triangular structures was placed between an optically transparent sapphire and a silica plate (Fig. 2a). Liquids were loaded into the chamber through microfluidic tubes and gas bubbles were created by incomplete filling of the liquid into the triangular cavities (Fig. 2b). The sample chamber was loaded onto a custom-built microscope (Materials and Methods and Supplementary Section 1) and a temperature gradient was generated by differentially heating the sapphire with rod resistors inserted into a copper holder and cooling the copper back-plate through a connection to a water bath (Fig. $2 \mathrm{c}, \mathrm{d})$. The temperature gradients were varied between 15 and $29^{\circ} \mathrm{C}$ with an accuracy of $\pm 1^{\circ} \mathrm{C}$. Imaging was provided through the transparent sapphire with the camera focused on the cold wall. This chamber is also referred to as a "thermal trap".

Coacervate microdroplet dispersions were prepared by mixing negatively charged modified sugars CM-Dex (degree of polymerization between 50 and 100, with 1 carboxyl group every 3 repeats) or ATP, with positively charged polyelectrolytes, either pLys (degree of polymerization of 20 to 70) or PDDA (degree of polymerization of 90) (Fig. 2e). CM-Dex:PDDA and CM-Dex:pLys mixtures were prepared at molar ratios of $6: 1$ and $4: 1$, respectively, whilst ATP:PDDA and ATP:pLys droplets were prepared at molar ratios of $4: 1$. The molar ratios correspond to a [carboxyl] to [amine] ratio of 5 (CM-Dex:PDDA) or 7 (CM-Dex:pLys). Such ratios were optimized in previous work to yield a good amount of coacervation ${ }^{11,30}$.
The total polymer concentrations were varied between 2 and $20 \mathrm{mM}$. The starting concentration dictated the density of coacervate droplets within the dispersion and the final amount of material accumulated at the gas-water interface. In order to visualize the coacervate droplets, we added $0.1 \%$ fluorescein isothiocyanate (FITC)-labelled CM-Dex or pLys. The coacervate dispersions were prepared in either $0.1 \mathrm{M} \mathrm{Na}^{+}$bicine buffer $(\mathrm{pH} 8.5)$ or $10 \mathrm{mM}$ Tris ( $\mathrm{pH}$ 8) and $4 \mathrm{mM} \mathrm{MgCl}_{2}$. Control experiments showed that there was no appreciable difference between the two different buffers regarding the dynamics of the coacervate within the thermal trap (Supplementary Section 2). Therefore, we used both buffers interchangeably throughout our experiments to highlight the generality of our findings.

On loading the coacervate dispersion (20 mM CM-Dex:PDDA in $0.1 \mathrm{M} \mathrm{Na}^{+}$bicine buffer, $\mathrm{pH} 8.5$ ) into the thermal trap, microscopy images (taken every $\sim 1 \mathrm{~s}$ ) showed the presence of small coacervate droplets $(<10 \mu \mathrm{m})$ evenly dispersed throughout the chamber (Fig. 3a). After differential heating at the two sides of the trap (warm side $49^{\circ} \mathrm{C}$, cold side $20^{\circ} \mathrm{C}$ ), the fluorescent droplets experienced convective flows in the bulk of the solution. The speed of the convective flow could be modulated by the temperature difference as observed in previous simulations ${ }^{27}$. Interestingly, we saw that the coacervate droplets in the bulk solution were transported by the convection flow to the gas-water interface where they accumulated and started growing by fusion (Fig. 3b,c and Supplementary Video 1). At the interface, the droplets moved parallel to the interface, driving contact and coalescence events. An individual fusion process between two coacervate droplets required a few seconds (from 1 to $10 \mathrm{~s}$ ) and resulted in elliptically shaped coacervates. Figure $3 \mathrm{~d}$ shows the process of fusion between three large coacervate droplets.

The growth of the coacervates over time was quantified from the optical microscopy images. Using LabVIEW, the average horizontal size was measured at different times (as depicted in Fig. 3b). Analysis showed that the CM-Dex:PDDA coacervates reached a maximal average size of $150 \mu \mathrm{m}$. Experiments with a different buffer $\left(10 \mathrm{mM}\right.$ Tris, $\mathrm{pH} 8$ and $\left.4 \mathrm{mM} \mathrm{MgCl}_{2}\right)$ or different polymers of different molecular weights (CM-Dex:pLys, ATP:pLys, ATP:PDDA or CM-Dex:pLys of higher molecular weight) showed comparable behaviour with minor differences in the final coacervate size (Fig. $3 e$ and Supplementary Section 2). Note that, in our analysis, we only measured the horizontal size and not the whole volume of the coacervate droplet. Therefore, we believe that our method was not sensitive to small changes in size. This could be why there was no particular observable effect of the buffer or coacervate type on the final droplet size. However, the method was successful in calculating the average size distribution, as shown in Figs. 3e and 5j.

In addition, we characterized the effect of total polymer concentration on the growth rate and the final size of the coacervate droplets by performing a series of experiments with a constant thermal gradient (warm side $49^{\circ} \mathrm{C}$ and cold side $20^{\circ} \mathrm{C}$ ), buffer conditions ( $4 \mathrm{mM} \mathrm{MgCl}_{2}, 10 \mathrm{mM}$ Tris, $\mathrm{pH} \mathrm{8.0)}$ and polymers (CM-Dex:PDDA molar ratio 6:1, [carboxyl]/[amine] $=5$ ), doped with $0.1 \%$ FITC-labelled CM-Dex. The total polymer concentration was varied between 1 and $20 \mathrm{mM}$ (a common concentration range that was used in other studies $\left.{ }^{12,18,31,32}\right)$. Immediately after inserting the coacervate solution in the thermal trap $(<1 \mathrm{~min})$, fluorescence microscopy images were taken every $\sim 1 \mathrm{~s}$. The images were analysed using ImageJ or LabVIEW to determine the growth rate and the average droplet size at steady state (after $1 \mathrm{~h}$ of thermal gradient) at the interface (Supplementary Section 3). The final droplet size and the growth rate did not seem to be significantly affected by the initial polymer concentration.

Also, we noticed that the variability in the size of the droplets between the experiments was large. This could be attributed to oscillatory salt fluctuations induced by the microscale water cycle, together with the intrinsic stochastic nature of droplet fusion. The 

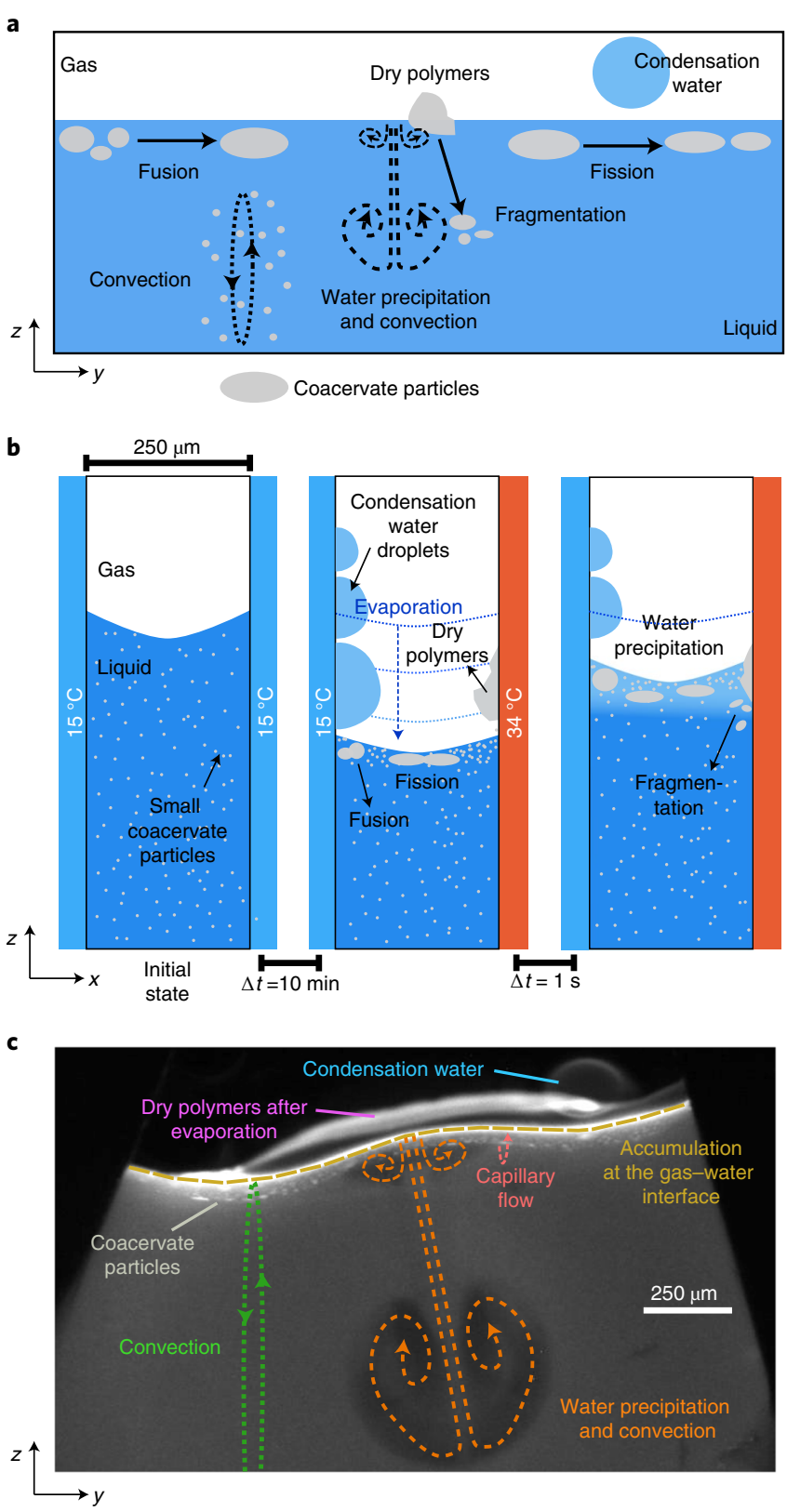

Fig. 1 | Fusion, division and transport of coacervate protocells inside a thermal pore. a, Scheme of coacervate transport, accumulation, growth and division at the gas-water interface, driven by convective flows, water condensation and subsequent water preciptation and convection. b. Left, scheme showing the thermophoretic pore in the absence of heating with pre-formed small coacervate droplets in the bulk. Centre, temperature gradient by differential heating across the pore with a gas bubble leads to water evaporation and a decrease in the water level that leads to dried polymers on the surface of the pore. Furthermore, droplet accumulation, fusion and fission are observed. Right, water precipitation drives coacervate fragmentation. c, Fluorescence image showing evaporation, water condensation, wet-dry cycles, convection and capillary flows at the gaswater interface of the thermal pore. Conditions for $\mathbf{c}$ were: CM-Dex:PDDA total polymer concentration $2 \mathrm{mM}$ (molar ratio 6:1, [carboxy]) [amine $]=5)+0.1 \%$ FITC-labelled CM-Dex, $10 \mathrm{mM} \mathrm{MgCl}, 10 \mathrm{mM}$ Tris, $\mathrm{pH}$ 8 , temperature gradient of $19^{\circ} \mathrm{C}$ (warm side $34^{\circ} \mathrm{C}$, cold side $15^{\circ} \mathrm{C}$ ).

salt fluctuations induced by microscale water cycles in our thermal trap were previously characterized and showed periodic salt oscillations and perturbative flows caused by water precipitation ${ }^{27}$.
While salts are known to have a major impact on coacervation ${ }^{33,34}$, the effects of the salt oscillations on the coacervate droplets in the thermal trap do not appear to adversely affect the droplet stability, as the droplets stay intact at the interface. It is possible that the small fluctuations in salt concentration at the interface can induce local changes in the surface charge of the droplets, influencing droplet fusion and droplet composition. However, it is clear that the droplets are stable under these salt conditions. We estimated a $\sim 1 \%$ change in the bulk salt concentration, accounting for the total volume within the pore versus the volume of water that takes part in precipitation. Therefore, the high variability in the sizes of the droplets and their composition that we observed during our analysis was likely to be due to the intrinsic stochastic nature of droplet fusion.

Despite this, in all instances (more than 50 different experiments that explored different coacervate conditions, starting concentrations and buffer conditions) we saw that the coacervate droplets accumulated and fused together, indicating that the accumulation, fusion and maintenance of the coacervate droplet at the gas-water interface are general phenomena driven by the forces in the thermal trap rather than the chemistry of the coacervate dispersion.

We also performed experiments with starting polymer concentrations below the critical coacervate concentration (CCC), $\sim 1 \mathrm{mM}$ for the CM-Dex:PDDA coacervate dispersions. At a starting concentration of 0.2 or $0.05 \mathrm{mM}$, no coacervate droplets were observed using optical microscopy within the resolution of our experiment, despite evident polymer up-concentration at the gas-water interface (Supplementary Section 3). Our results indicate that the thermal pore acts at the mechanical level to drive fusion of previously existing coacervate droplets, followed by droplet division by stretching or fragmentation and aggregation by wet-dry cycling.

We then wanted to verify that these observed phenomena were attributed to the gas-water interface in combination with thermal flows. To this end, we undertook two control experiments. The first determined the effect of convective flow alone, that is, in the absence of a gas bubble on the coacervate droplets. To do this, coacervate dispersions (CM-Dex:pLys, $2 \mathrm{mM}$, ratio 4:1, [carboxyl $] /[$ amine $]=7,10 \mathrm{mM}$ Tris, $\mathrm{pH} 8.0,4 \mathrm{mM} \mathrm{MgCl}_{2}$ ) were loaded into a thermal trap without gas bubbles (warm side $49^{\circ} \mathrm{C}$, cold side $20^{\circ} \mathrm{C}$ ). Time-resolved optical microscopy images showed that the bulk coacervate droplets $(<15 \mu \mathrm{m})$ were transported in the bulk by the convection flow at a speed of about $1.6 \pm 0.4 \mu \mathrm{m} \mathrm{s}^{-1}$ but did not undergo fusion events in the bulk solution or accumulate within the trap (Supplementary Section 4). We then characterized the behaviour of coacervate droplets within the thermal chamber in the absence of thermal flow. At isothermal conditions, almost $100 \%$ of coacervate droplets within the pore slowly sedimented to the bottom of the microfluidic chamber, where the droplets fused to form a single coacervate droplet, as expected under isothermal conditions ${ }^{18,19}$. In the presence of the thermal gradient, the convection flow in the bulk prevented the coacervate droplets from sedimenting by maintaining them within the thermophoretic flow. The fraction of droplets that survived sedimentation was proportionally dependent on the thermal gradient. Steeper thermal gradients induced faster convection and prevented the sedimentation of a larger fraction of droplets. Finite element simulations of the sedimentation of the coacervate droplets in a thermal trap, with comparable thermal gradients to the experiments, showed that droplet sedimentation reached a steady state after $5 \mathrm{~h}$ and this was maintained for up to $30 \mathrm{~h}$ (Supplementary Section 5). In comparison, coacervate droplets at the gas-water interface resided at the interface even with very shallow temperature gradients.

Taken together, our results confirm that the flows at a gas-water interface led to the accumulation of coacervate droplets at the interface, fusion events between the droplets and to the maintenance of the droplets against sedimentation. In the absence of the thermal flow, the droplets will sediment to the bottom of the pore. Therefore, 


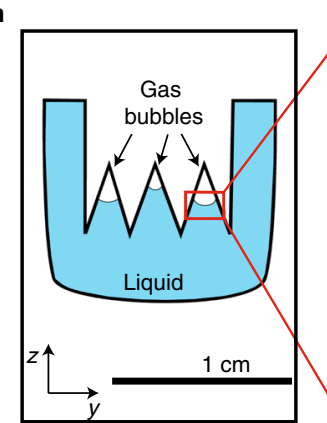

c
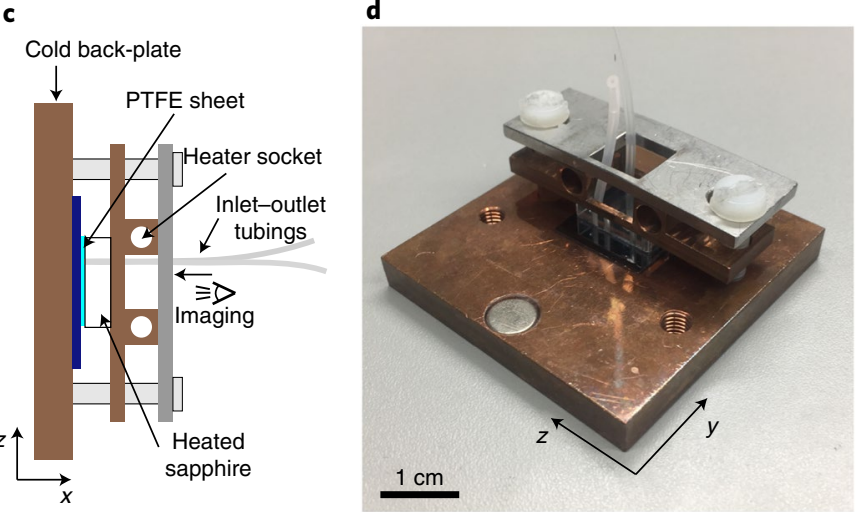

e PDDA

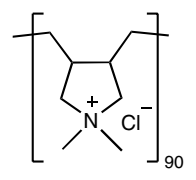

b

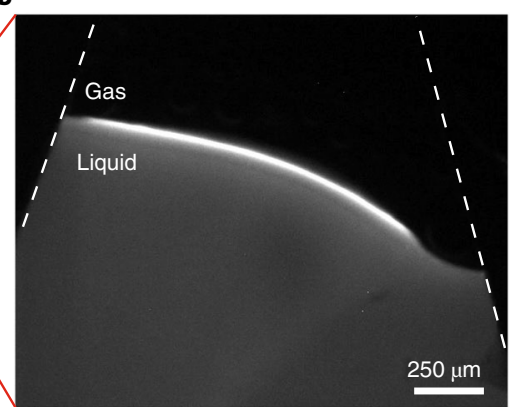

CM-Dex

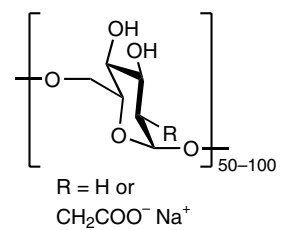

$\mathrm{CH}_{2} \mathrm{COO}^{-} \mathrm{Na}^{+}$

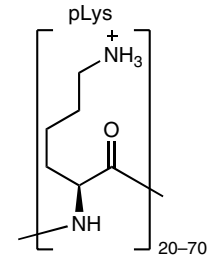

ATP

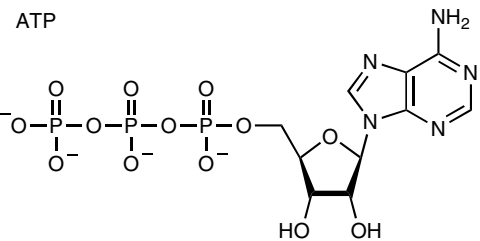

RNA (51 nt)

5' - UUA GCA GAG CGA GGU AUG T ${ }^{\text {ROX }}$ AG GCG GGA CGC UCA GUG GAA CGA AAA CUC ACG -3'

Fig. 2 | Description of the thermal trap used in the experiments. $a$, Schematic of the PTFE interspacer. The triangular structures cause the formation of gas bubbles on addition of buffer. $\mathbf{b}$, Fluorescence image of the gas bubble and gas-liquid interface in a thermal trap with temperature gradient (CM-Dex:PDDA 6:1 ratio + 0.1\% FITC-labelled CM-Dex, total concentration $2 \mathrm{mM}$ ). c,d, Lateral sketch (c) and photograph (d) of the thermal trap. The PTFE sheet with defined geometry was placed between a transparent sapphire and a cold copper back-plate. The sapphire was heated with rod resistors whilst the copper back-plate was cooled using a water bath to generate a temperature gradient. Aqueous solution was loaded and removed from the chamber by inlet and outlet tubings. e, Chemical structures of the components used: PDDA, pLys, CM-Dex, ATP, RNA sequence (51 nucleotides ( $n t)$ ).

the combination of convection and capillary flow at the interface maintained the droplets at the gas-water interface or circulating within the bulk for extended periods of time.

Droplet division at the gas-water interface. Our data show that the opposing forces at the interface lead to the elongation of the droplets (Fig. 3c,d). As an elliptical shape has been associated with the initial stages of vesicle division ${ }^{35}$ we wondered whether the forces in our non-equilibrium setting would be strong enough to drive the elliptical deformation of the membrane-free coacervate droplet into a fission event.

We applied a temperature gradient of $19 \mathrm{~K}\left(15-34^{\circ} \mathrm{C}\right)$ on a coacervate dispersion of CM-Dex:PDDA (molar ratio 6:1, [carboxyl $] /[$ amine $]=5$, total polymer concentration $2 \mathrm{mM}, 10 \mathrm{mM}$ Tris, $\mathrm{pH} 8,4 \mathrm{mM} \mathrm{MgCl}_{2}$ ) doped with $0.1 \%$ FITC-labelled CM-Dex. Time-resolved optical microscopy images show that the coacervate droplets accumulated, fused and became elliptically elongated at the gas-water interface (Fig. 3c,d). Excitingly, on accumulation, droplets were progressively stretched along the interface until the droplet divided to produce two daughter protocells of a similar size (Fig. 4a and Supplementary Video 2). Our results confirm that elliptical deformation of the coacervate droplets at the interface do indeed drive droplet division. Droplet stretching and fission occurred as a consequence of the forces induced by the thermal gradient at the gas-water interface. In additional experiments, CM-Dex:pLys droplets also underwent fission events at the interface, indicating that this is a general phenomenon that is driven by the physical forces rather than the chemistry or type of coacervate (Supplementary Section 6).

In addition to convection and capillary forces at the interface, the presence of a gas bubble creates an environmental water cycle-this hypothetical prebiotic scenario may also have an effect on coacervate behaviour and properties. For example, wet-dry cycles can lead to the accumulation, drying and rehydration of molecules at a surface. Previous studies ${ }^{27,28}$ have shown that a heated gas bubble in contact with a cold surface within a thermal trap will simulate a microfluidic water cycle. Pure water from the bulk solution will evaporate at the warm side and condense on the cold surface. These water droplets will grow in size and fall back into solution. The evaporation, water condensation and re-entry into the bulk solution leads to decrease (evaporation) and increase (rainfall) of the interface height. We therefore sought to determine how such wet-dry cycles and water precipitation would affect the coacervate droplets.

To do this, a dispersion of coacervate microdroplets (CM-Dex:PDDA, molar ratio 6:1, [carboxyl]/[amine] =5, total polymer concentration $20 \mathrm{mM}, 10 \mathrm{mM}$ Tris, $\mathrm{pH} 8,4 \mathrm{mM} \mathrm{MgCl}_{2}$, doped with $0.1 \%$ FITC-labelled CM-Dex) was loaded into the thermal trap with a temperature gradient (warm side $34^{\circ} \mathrm{C}$, cold side $15^{\circ} \mathrm{C}$ ). Time-resolved optical microscopy images (Fig. $4 \mathrm{~b}$ and Supplementary Video 3) show that coacervate droplets accumulated at the gas-water interface and stuck to the warm surface of the trap as the height of the interface decreased from water evaporation. This had the effect of driving the accumulated coacervates into a quasi-dry state on the surface. The dry polymers (see arrow in Fig. 4 b) were later rehydrated and the perturbative fluxes induced by the water precipitation led to their fragmentation. The resulting smaller daughter droplets fell into the bulk and circulated with the convection flow. These results show that water cycles can drive the fragmentation and fission of coacervate droplets. Again, additional experiments with CM-Dex:pLys mixtures showed that this process is general and can also take place when different types of coacervate are used (Supplementary Section 6).

Despite this, fission events were rarely observed. Out of a total of 53 experiments (average duration of $\sim 2 \mathrm{~h}$ each) which explored different polymer types, polymer concentrations, temperature gradients, buffers and trap geometries, we observed 12 division events. Of these 12 events, 10 of them consisted of division by fragmentation (the type of Fig. 4b). Two of them were of the type shown in Fig. 4a. However, the division events may be happening more frequently since we only image one of the many gas bubbles that were present 

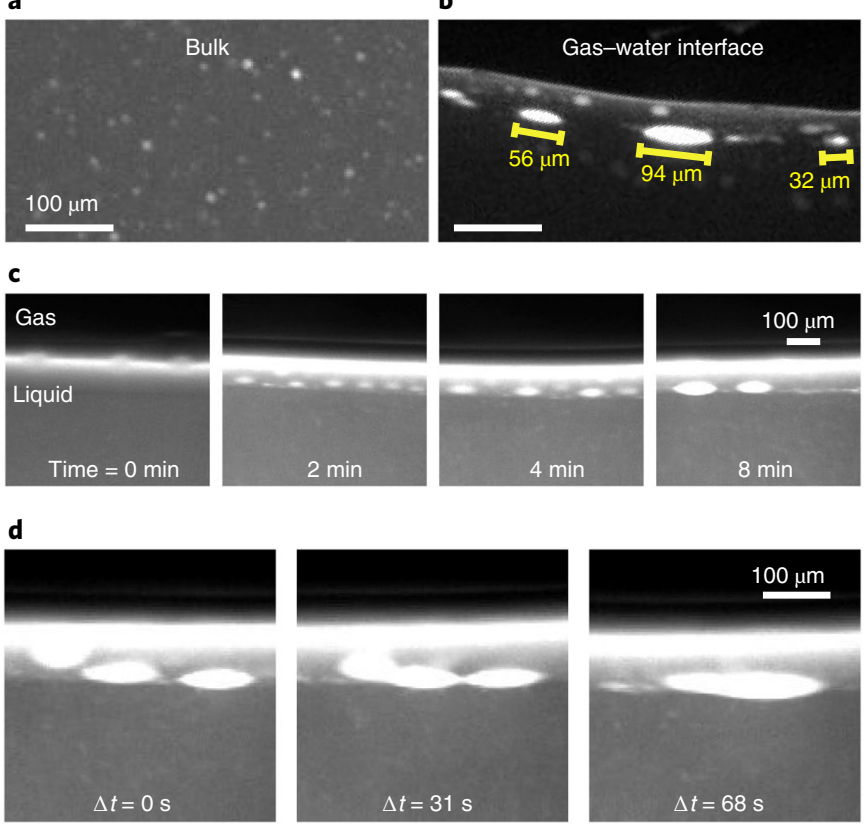

- CM-Dex:PDDA, $0.1 \mathrm{M} \mathrm{Na}^{+}$bicine

- CM-Dex:PDDA, $4 \mathrm{mM} \mathrm{MgCl} 2$ and $10 \mathrm{mM}$ Tris

CM-Dex:pLys, $4 \mathrm{mM} \mathrm{MgCl} 2$ and $10 \mathrm{mM}$ Tris

- ATP:pLys, $4 \mathrm{mM} \mathrm{MgCl}_{2}$ and $10 \mathrm{mM}$ Tris

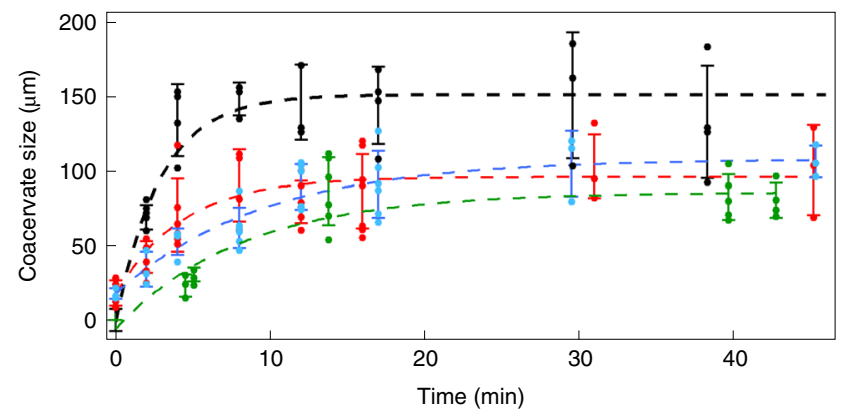

Fig. 3 | Coacervate droplets accumulate and fuse at the gas-water interface. $\mathbf{a}, \mathbf{b}$, Fluorescence microscopy images of coacervate droplets in bulk (a) and at the gas-water interface (b). On implementation of the thermal gradient, convective flows transport the coacervate droplets in the bulk to the gas-water interface where they fuse. Droplets will grow until they reach a steady-state size which is then maintained over time. c, Coacervate droplets at the interface (from left to right) at $t=0,2,4$ and $8 \mathrm{~min}$ in a thermal gradient show progressive increase in droplet size. $\mathbf{d}$, Microscopy images showing a fusion event between three coacervate droplets. e, Quantification of coacervate size over time for different buffer and coacervate compositions. Each data point represents the mean and standard deviation of approximately five different larger droplets at the gas-water interface. The dashed lines represent phenomenological exponential fits.

in the chamber. It is also important to note that our imaging protocol projected the view of the thermal trap on a $2 \mathrm{D}$ plane and was therefore not able to distinguish objects or observe any dynamics in the perpendicular axis. In Supplementary Section 7, we thoroughly analysed the experiment shown in Fig. 4a to rule out possible artifacts derived from the imaging.

Taken together, our results show two mechanisms by which the out-of-equilibrium behaviour induced by the thermal gradient at the gas-water interface of a microfluidic pore can drive droplet fission. This represents a viable route to coacervate fission and subsequent evolution within the prebiotically plausible scenario of a thermal pore.

Furthermore, to determine how robust the behaviour within the pore was, we characterized the effect of different temperature gradients (values of $\Delta T$ between 10 and $60 \mathrm{~K}$ ), trap thicknesses (between 127 and $500 \mu \mathrm{m}$ ) and the volume of the gas bubbles (between 0.005 and $50 \mathrm{~mm}^{3}$ ) on dispersions of coacervate droplets. Within these broad ranges of conditions, the features of coacervate accumulation, fusion, wet-dry cycles and divisions were observed. It appears that differences in these three parameters can affect the sedimentation and accumulation properties, fusion and division events and the quantity of dried polymers on the surface of the pore. For example, steep temperature gradients induce a fast convection in the bulk which prevents sedimentation and induces a fast capillary flow that promotes the fusion between the droplets. The increased wet-dry cycles also promote the division mechanism by fragmentation (Fig. $4 \mathrm{~b})$. On the other hand, droplet division by stretching would benefit from shallower temperature gradients, because the droplet needs to be slowly stretched in order to divide (Fig. 4a). In addition, steep temperature gradients will affect the size and frequency of water precipitations and, consequently, the extent to which the gas-water interface moves up and down during the evaporation/water condensation cycles that affect the quantity of dried polymers.

In summary, the general properties of accumulation, fusion and division, drying and coacervate re-entry are observed across a broad range of experimental conditions such as the temperature gradient, the chamber thickness and the gas-to-liquid ratio. Tuning these experimental parameters will tune the dynamic behaviour of the droplets in the pore. This provides exciting and plausible evidence that our observed phenomena of flow-induced droplet maintenance, accumulation, fusion and fission could have taken place within rocky environments of early Earth, which had pores of different sizes, incorporated bubbles of different dimensions and were subject to different thermal gradients.

Separation and selection of coacervate phenotypes. So far, we have determined the effect of the thermal trap with gas bubbles on coacervates prepared from modified sugars, peptides and synthetic polymers. Despite the fact that PDDA was unlikely as a prebiotic molecule, we observed the general phenomena of accumulation, fusion, maintenance and fission by different mechanisms which appear independent of the chemical properties of the coacervate (Supplementary Fig. 2).

Recent studies have shown that compartmentalization by coacervation $^{12,36}$ or the hydrophobic effect with fatty acids ${ }^{37}$ could complement the RNA world hypothesis by providing the means to accumulate RNA and regulate RNA activity. Therefore, we wanted to determine the effect of the out-of-equilibrium dynamics of the thermal trap on dispersions of CM-Dex, pLys and RNA. To do this, dispersions of CM-Dex and pLys (molar ratio 4:1, [carboxyl]/ [amine] =7) with and without RNA (51 nt, single-stranded, Fig. 2e) were prepared at concentrations of $1.5 \mathrm{mM}, 0.5 \mathrm{mM}$ and from $0-5 \mu \mathrm{M}$ respectively in $10 \mathrm{mM}$ Tris, $\mathrm{pH} 8$ and $4 \mathrm{mM} \mathrm{MgCl}_{2}$. In order to study the co-localization between RNA, CM-Dex and pLys, dual-channel fluorescence imaging was used. RNA was labelled with ROX (carboxy-X-rhodamine) while $0.1 \%$ of the coacervate components (CM-Dex or pLys) contained a FITC label (see Fig. 5a). The microscope was equipped with an image splitter (Optosplit II) containing the filterset for FITC and ROX to enable dual-channel fluorescence imaging.

After loading the dispersions of CM-Dex and pLys with RNA into the sample chamber, dual-channel fluorescence imaging showed that pre-formed small coacervate droplets ( colocalized RNA. Microscopy images showed that already prior to the thermal gradient, the droplets were rich in RNA and pLys with a weak signal attributed to CM-Dex. This indicates that RNA strongly 

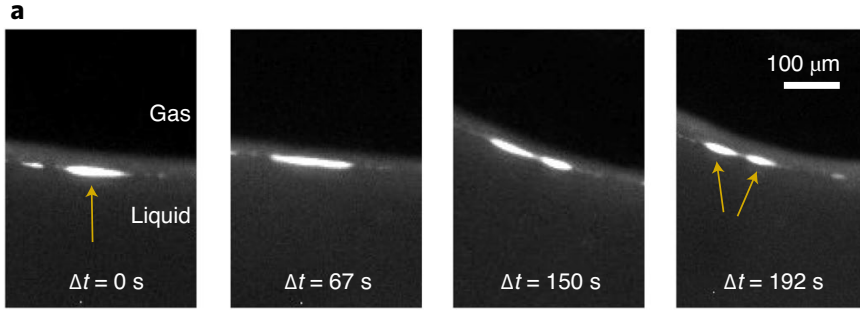

b
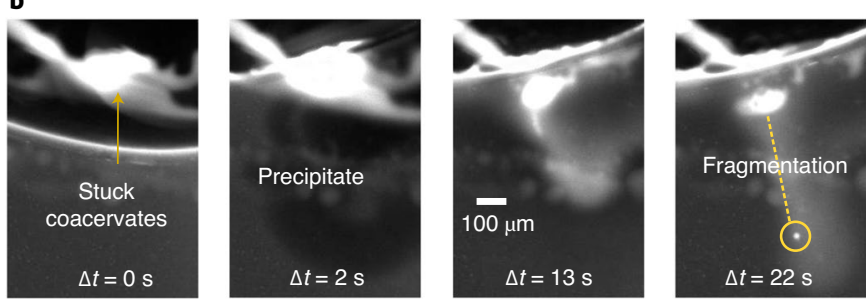

Fig. 4 | Fission of coacervates induced by interfacial forces and fluxes caused by water precipitation. a, Fission of a coacervate droplet into two smaller droplets, induced by interfacial forces at the gas-liquid interface. The initial droplet (yellow arrow) is slowly stretched (over a time frame of minutes) at the interface until it divides into two smaller droplets. $\mathbf{b}$, Rehydration of coacervates stuck to the surface can induce fission by fragmentation, due to the perturbative fluxes caused by precipitating water. It induces a fast mixing of the dry polymers that eventually fragment.

competes with CM-Dex to form droplets with pLys. Indeed, thermophoretic measurements to obtain the binding constants between RNA with pLys and CM-Dex with pLys confirmed a higher affinity of RNA for pLys compared to CM-Dex (Supplementary Section 8). Fitting to the dose-response curve, we found that the dissociation constant $\left(K_{\mathrm{D}}\right)$ of the RNA:pLys complex $\left(K_{\mathrm{D}}<11 \mathrm{nM}\right)$ is an order of magnitude lower than the $K_{\mathrm{D}}$ of the CM-Dex:pLys complex $\left(120 \mathrm{nM}<K_{\mathrm{D}}<400 \mathrm{nM}\right)$. This difference in $K_{\mathrm{D}}$ may be attributed to the fact that RNA has a higher charge density compared to CM-Dex. Therefore, whilst there is a small amount of CM-Dex within the droplet, CM-Dex will also be free in the coacervate dispersion. On inducing a thermal gradient (warm side $34^{\circ} \mathrm{C}$, cold side $15^{\circ} \mathrm{C}$ ), we observed the same phenomena as described previously, that is that coacervate droplets accumulate at the interface and fuse together. Interestingly, with the three coacervate components, dual fluorescence imaging of dispersions containing either FITC-labelled CM-Dex or pLys, with ROX-labelled RNA showed that the droplets at the interface were larger and contained all three components (CM:Dex, RNA and pLys) (Fig. 5a-c and Supplementary Video 4) whilst the droplets in the bulk remained small and rich in RNA and pLys (Fig. 5d-f). This observation is most likely to be due to the ability of the thermal trap to drive a strong accumulation of the RNA, pLys and CM-Dex in solution to the gas-water interface and induce an enrichment of the three components within the coacervate droplets, overcoming the equilibrium binding constants (Supplementary Section 8). Merging of the optical images shows that the microdroplets in the bulk have an overlap of the fluorescence signals of RNA and pLys (Fig. 5d-f and Supplementary Section 9).

These results are important as they show that the thermal pore can generate and select for two different populations of coacervate droplets with different chemical compositions at the gas-water interface and within the bulk solution, which has not been previously reported on.

We quantified the droplet size at the interface after applying the thermal gradient for $1 \mathrm{~h}$ using the methodologies already described and as a function of RNA concentration. We observed that the final size of the coacervate protocells at the gas-water interface was inversely affected by RNA. In the presence of RNA, the average coacervate size dropped from $69 \pm 31 \mu \mathrm{m}$ down to $25 \pm 9 \mu \mathrm{m}$ (Fig. $5 g-j$ and Supplementary Figure S8). As already shown in other studies $^{38}$, a higher charge density can lead to the formation of smaller coacervate droplets. This is in fact what we observed and we believe that this effect is driven by the stronger binding of RNA to pLys compared to CM-Dex to pLys.

The results show how the thermal trap can keep the coacervate droplets in a non-equilibrium state, enabling energetically unfavourable interactions at the interface. This permits the formation and selection of two different populations of droplets within the pore with different physical properties and different compositions. The results also show that the chemical composition of the coacervate droplets will affect their phenotype with smaller droplet size for increasing RNA concentration.

\section{Discussion}

We have shown that experimental primordial conditions-a millimetre-sized pore in a temperature gradient with a gas bubble-imparted specific selection pressures on dispersions of coacervate microdroplets. The thermal gradient across the pore drove a convection flow within the bulk solution and instigated the accumulation and growth of the coacervate droplets by fusion at the gas-water interface. The forces in the heated rock-like pores hindered the sedimentation of the coacervate droplets and the formation of large coacervate macrophases whilst permitting the maintenance of cell sized coacervate microdroplets for longer times. These droplets were elongated due to convection and capillary forces and underwent division after deformation at the gaswater interface. In addition, we observed division as a consequence of a water cycle within the gas bubble. The water precipitations induced the division and fragmentation of the coacervate material accumulated on the surface of the pore. These features were not observed in thermal traps in the absence of gas bubbles or at isothermal temperatures, indicating that this was a unique property of the thermal gradient and the gas bubble. This is a clear example of the accumulation, fusion, maintenance and fission of coacervate protocells. We have shown that this is a general phenomenon as we observed the same processes in coacervates with different chemical compositions and buffer conditions. These results represent a possible mechanism for the growth and division of membrane-free protocells on primordial Earth.

We have also shown that $K_{\mathrm{D}}$ determined the affinity of polyelectrolytes to form coacervates where oligonucleotides (RNA) had a higher propensity to form coacervates with polypeptides (pLys) compared to modified sugars (CM-Dex). The coacervate microdroplets that we studied seemed to be selective towards RNA (a molecule which can be catalytic) incorporation. In an origin-of-life scenario, this process could give a selective advantage in terms of catalysis within a pool of coacervate protocells. The thermal trap generated two different populations of coacervate droplets, where droplets poor in CM-Dex were maintained in the bulk solution whilst CM-Dex rich droplets formed and accumulated at the gas-water interface. This finding shows that the environment of a thermal trap with a gas bubble enables energetically unfavourable coacervate droplets to form by driving the system into an out-of-equilibrium state. As a consequence, the thermal trap was able to generate and contain populations of coacervate droplets which differ in chemical composition and size and therefore physical properties. In the presence of active RNA, these genotypic and phenotypic differences would be most likely to lead to different activities within the droplet. The droplets at the gas-water interface would benefit from additional variability and non-equilibrium properties: preferential enrichment of longer oligonucleotides ${ }^{28}$, enhanced strand separation at lower temperatures ${ }^{27}$ (and therefore lower hydrolysis rates) and enhanced RNA catalysis induced by the presence of an 

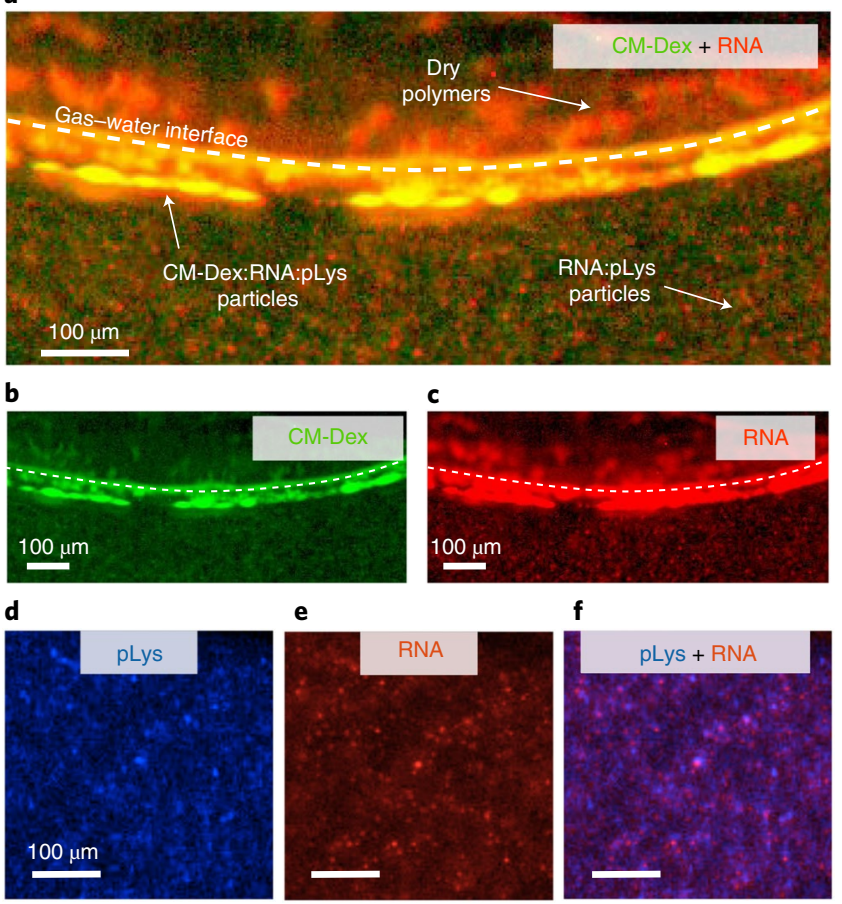

e

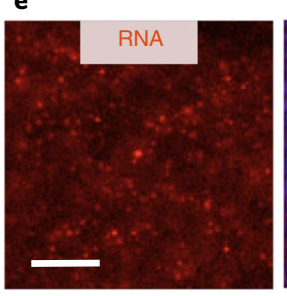

g

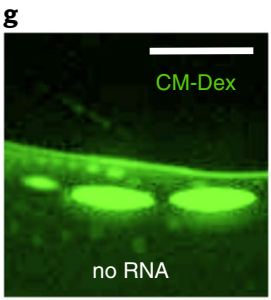

h

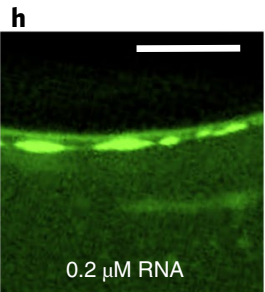

i
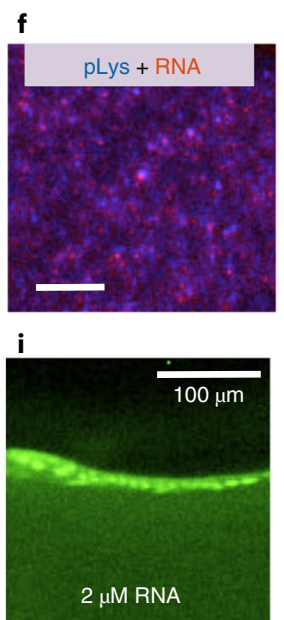

j

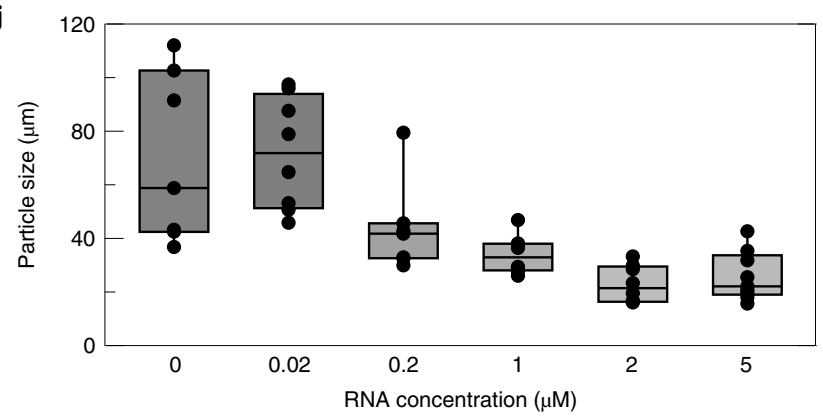

Fig. 5 | The thermal trap creates and separates two populations of coacervate droplets. a-c, Dual-channel fluorescence images of the CM-Dex:pLys:RNA coacervates in the thermal trap. CM-Dex and RNA were differentially labelled with FITC $0.1 \%$ and ROX $100 \%$, respectively. The single pictures of the composite (a) are shown in $\mathbf{b}$ and $\mathbf{c}$, respectively. Small droplets ( $<15 \mu \mathrm{m})$ enriched in RNA and pLys are formed in the bulk. Droplets enriched of all three components form instead at the gas-water interface. d-f, pLys channel (0.1\% FITC-labelled) (d), RNA channel (e) and composite image (f), showing co-localization between RNA and plys in the bulk droplets. $\mathbf{g}$-i, no RNA (g), $0.2 \mu \mathrm{M}$ RNA (h) and $2 \mu \mathrm{M}$ RNA (i) showing the droplets at the gas-water interface (CM-Dex channel). j, Quantification of the size of CM-Dex:pLys droplets as a function of RNA concentration. The bars indicate the average size and standard deviation of nine different coacervate droplets.

additional polyanionic component that could lead to a change in material properties and the diffusion and reaction rates of RNA within the coacervate ${ }^{39}$.
This has important implications for demonstrating how thermal fluxes could have driven an evolutionary selection pressure on coacervate microdroplets, giving experimental evidence for a key role within the origin-of-life scenario. In conclusion, our work has shown that a temperature gradient with a gas bubble generates a unique environment for the accumulation, fusion, fission and selection of coacervate microdroplets. We have shown that these characteristics have been made accessible by physical forces alone, without the chemical complexity or sophisticated machinery seen in modern biology. This makes the gas bubble within a heated rock pore a compelling scenario to drive the evolution of membrane-free coacervate microdroplets on early Earth.

\section{Online content}

Any methods, additional references, Nature Research reporting summaries, source data, extended data, supplementary information, acknowledgements, peer review information; details of author contributions and competing interests; and statements of data and code availability are available at https://doi.org/10.1038/ s41557-021-00830-y.

Received: 25 November 2020; Accepted: 30 September 2021; Published online: 6 December 2021

\section{References}

1. Mason, A. F. et al. Mimicking cellular compartmentalization in a hierarchical protocell through spontaneous spatial organization. ACS Cent. Sci. 5, 1360-1365 (2019).

2. Sokolova, E. et al. Enhanced transcription rates in membrane-free protocells formed by coacervation of cell lysate. Proc. Natl Acad. Sci. USA 110, 11692-11697 (2013).

3. Oparin, A. I. The Origin of Life 2nd edn (Dover Publications, 1953).

4. Priftis, D., Laugel, N. \& Tirrell, M. Thermodynamic characterization of polypeptide complex coacervation. Langmuir 28, 15947-15957 (2012).

5. Koga, S., Williams, D. S., Perriman, A. W. \& Mann, S. Peptide-nucleotide microdroplets as a step towards a membrane-free protocell model. Nat. Chem. 3, 720-724 (2011).

6. Tena-Solsona, M. et al. Kinetic Control over droplet ripening in fuel-driven active emulsions. Preprint at ChemRxiv https://doi.org/10.26434/ CHEMRXIV.9978539.V1 (2019).

7. Crosby, J. et al. Stabilization and enhanced reactivity of actinorhodin polyketide synthase minimal complex in polymer-nucleotide coacervate droplets. Chem. Commun. 48, 11832 (2012).

8. McCall, P. M. et al. Partitioning and enhanced self-assembly of actin in polypeptide coacervates. Biophys. J. 114, 1636-1645 (2018).

9. Nakashima, K. K., Vibhute, M. A. \& Spruijt, E. Biomolecular chemistry in liquid phase separated compartments. Front. Mol. Biosci. 6, 1-9 (2019).

10. Beneyton, T., Love, C., Girault, M., Tang, T. -Y. D. \& Baret, J. Highthroughput synthesis and screening of functional coacervates using microfluidics. ChemSystemsChem 2, e2000022 (2020).

11. Tang, T.-Y. D. et al. Fatty acid membrane assembly on coacervate microdroplets as a step towards a hybrid protocell model. Nat. Chem. 6, 527-533 (2014).

12. Drobot, B. et al. Compartmentalised RNA catalysis in membrane-free coacervate protocells. Nat. Commun. 9, 3643 (2018).

13. Poudyal, R. R. et al. Template-directed RNA polymerization and enhanced ribozyme catalysis inside membraneless compartments formed by coacervates. Nat. Commun. 10, 490 (2019).

14. Poudyal, R. R., Pir Cakmak, F., Keating, C. D. \& Bevilacqua, P. C. Physical principles and extant biology reveal roles for RNA-containing membraneless compartments in origins of life chemistry. Biochemistry 57, 2509-2519 (2018).

15. Santos, M., Zintzaras, E. \& Szathmáry, E. Origin of sex revisited. Origins Life Evol. Biospheres 33, 405-432 (2003).

16. Hanczyc, M. M. \& Szostak, J. W. Replicating vesicles as models of primitive cell growth and division. Curr. Opin. Chem. Biol. 8, 660-664 (2004).

17. Smith, A. E. \& Chance, M. A. C. Coacervate behaviour in an alternating electric field. Nature 209, 74-75 (1966).

18. Nakashima, K. K., Baaij, J. F. \& Spruijt, E. Reversible generation of coacervate droplets in an enzymatic network. Soft Matter 14, 361-367 (2018).

19. Mason, A. F., Buddingh, B. C., Williams, D. S. \& Van Hest, J. C. M. Hierarchical self-assembly of a copolymer-stabilized coacervate protocell. J. Am. Chem. Soc. 139, 17309-17312 (2017).

20. Schafer, K. A. The cell cycle: a review. Vet. Pathol. 35, 461-478 (1998). 
21. Beltrán-Heredia, E., Almendro-Vedia, V. G., Monroy, F. \& Cao, F. J. Modeling the mechanics of cell division: influence of spontaneous membrane curvature, surface tension, and osmotic pressure. Front. Physiol. 8, 312 (2017).

22. Zhu, T. F., Adamala, K., Zhang, N. \& Szostak, J. W. Photochemically driven redox chemistry induces protocell membrane pearling and division. Proc. Natl Acad. Sci. USA 109, 9828-9832 (2012).

23. Kudella, P. W. et al. Fission of lipid-vesicles by membrane phase transitions in thermal convection.Sci. Rep. 9, 18808 (2019).

24. Pal, A. \& Khakhar, D. V. Breakage of vesicles in a simple shear flow. Soft Matter 15, 1979-1987 (2019).

25. Zwicker, D., Seyboldt, R., Weber, C. A., Hyman, A. A. \& Jülicher, F. Growth and division of active droplets provides a model for protocells. Nat. Phys. 13 , 408-413 (2017).

26. Agerschou, E. D., Mast, C. B. \& Braun, D. Emergence of life from trapped nucleotides? Non-equilibrium behavior of oligonucleotides in thermal gradients. Synlett 28, 56-63 (2017).

27. Ianeselli, A., Mast, C. B. \& Braun, D. Periodic melting of oligonucleotides by oscillating salt concentrations triggered by microscale water cycles inside heated rock pores. Angew. Chem. Int. Ed. 58, 13155-13160 (2019).

28. Morasch, M. et al. Heated gas bubbles enrich, crystallize, dry, phosphorylate and encapsulate prebiotic molecules. Nat. Chem. 11, 779-788 (2019).

29. Ukmar-Godec, T. et al. Lysine/RNA-interactions drive and regulate biomolecular condensation. Nat. Commun. 10, 2909 (2019).

30. Moreau, N. G., Martin, N., Gobbo, P., Tang, T. Y. D. \& Mann, S. Spontaneous membrane-less multi-compartmentalization: via aqueous two-phase separation in complex coacervate micro-droplets. Chem. Commun. 56, 12717-12720 (2020).

31. Donau, C. et al. Active coacervate droplets as a model for membraneless organelles and protocells. Nat. Commun. 11, 5167 (2020).

32. Love, C. et al. Reversible $\mathrm{pH}$-responsive coacervate formation in lipid vesicles activates dormant enzymatic reactions. Angew. Chem. Int. Ed. 59, 5950-5957 (2020)
33. Van Der Kooij, H. M. et al. On the stability and morphology of complex coacervate core micelles: from spherical to wormlike micelles. Langmuir $\mathbf{2 8}$, 14180-14191 (2012).

34. Perry, S., Li, Y., Priftis, D., Leon, L. \& Tirrell, M. The effect of salt on the complex coacervation of vinyl polyelectrolytes. Polymers (Basel) 6 , 1756-1772 (2014).

35. Urakami, N., Jimbo, T., Sakuma, Y. \& Imai, M. Molecular mechanism of vesicle division induced by coupling between lipid geometry and membrane curvatures. Soft Matter 14, 3018-3027 (2018).

36. Mountain, G. A. \& Keating, C. D. Formation of multiphase complex coacervates and partitioning of biomolecules within them. Biomacromolecules 21, 630-640 (2020).

37. Cornell, C. E. et al. Prebiotic amino acids bind to and stabilize prebiotic fatty acid membranes. Proc. Natl Acad. Sci. USA 116, 17239-17244 (2019).

38. Vieregg, J. R. et al. Oligonucleotide-peptide complexes: phase control by hybridization. J. Am. Chem. Soc. 140, 1632-1638 (2018).

39. Poudyal, R. R., Keating, C. D. \& Bevilacqua, P. C. Polyanion-assisted ribozyme catalysis inside complex coacervates. ACS Chem. Biol. 14, 1243-1248 (2019)

Publisher's note Springer Nature remains neutral with regard to jurisdictional claims in published maps and institutional affiliations.

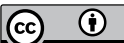

Open Access This article is licensed under a Creative Commons

Attribution 4.0 International License, which permits use, sharing, adaptation, distribution and reproduction in any medium or format, as long as you give appropriate credit to the original author(s) and the source, provide a link to the Creative Commons license, and indicate if changes were made. The images or other third party material in this article are included in the article's Creative Commons license, unless indicated otherwise in a credit line to the material. If material is not included in the article's Creative Commons license and your intended use is not permitted by statutory regulation or exceeds the permitted use, you will need to obtain permission directly from the copyright holder. To view a copy of this license, visit http://creativecommons.

org/licenses/by/4.0/.

(C) The Author(s) 202 


\section{Methods}

CM-Dex sodium salt (10-20 or 150-300 kDa, monomer: $191.3 \mathrm{~g} \mathrm{~mol}^{-1}$ ), pLys hydrobromide (4-15 or $150 \mathrm{kDa}$, monomer: $208.1 \mathrm{~g} \mathrm{~mol}^{-1}$ ) and PDDA (8.5 kDa, monomer: $\left.161.5 \mathrm{~g} \mathrm{~mol}^{-1}\right)$, FITC-labelled pLys (15-30 kDa), FITC-labelled CM-Dex $(15$ or $150 \mathrm{kDa})$ and ATP $\left(507.2 \mathrm{~g} \mathrm{~mol}^{-1}\right)$ were purchased from Sigma-Aldrich and were used without further purification. Stock solutions of each of the coacervate components were prepared to a concentration of $1 \mathrm{M}$ in MilliQ water and stored at $-20^{\circ} \mathrm{C}$ until use. RNA oligonucleotides were purchased from biomers.net $\mathrm{Gmbh}$, with HPLC purification and re-dissolved to a final concentration of $100 \mu \mathrm{M}$ in nuclease-free water. The sequence was (51 bases): 5' -UUA GCA GAG CGA GGU AUG ${ }^{\text {Rox }}$ AG GCG GGA CGC UCA GUG GAA CGA AAA CUC ACG-3’. Every RNA strand was labelled with a ROX molecule positioned centrally in the sequence attached to the backbone of a thymine and stored in pure nuclease-free water at a concentration of $100 \mu \mathrm{M}$.

The experiments were performed in a thin layer of PTFE $(250 \mu \mathrm{m})$, which was cut with a defined geometry and then placed between a transparent sapphire and a copper back-plate. The geometry of the PTFE sheet was designed to induce the incorporation of gas bubbles as shown in previous work ${ }^{27,28}$. The sapphire was in contact with a copper placeholder which was heated with rod resistors. The copper back-plate was attached to an aluminium holder which was cooled with liquid water from a water bath (300F from JULABO). Temperature sensors (GNTP-SG from Thermofühler $\mathrm{GmbH}$ ) were attached to the copper back-plate and to the copper sapphire holder to measure the outer temperatures of the cold and warm sides. The inner temperatures of the chamber were then calculated numerically, based on the outer temperatures, the heat conductivities of the materials (copper, silicon and sapphire) and their thickness. The outer warm target temperature was maintained constant via a PID loop implemented in LabVIEW, in order to control the output voltage to the rod resistors. The accuracy of the target temperatures was $\pm 1^{\circ} \mathrm{C}$. The temperature differences that we used in the experiments shown here ranged from 15 to $30^{\circ} \mathrm{C}$.

Coacervate components were mixed together to the final desired concentration $(2-20 \mathrm{mM})$ and immediately loaded into the microfluidic chamber. Dispersions of coacervates were prepared from either CM-Dex:PDDA or CM-Dex:pLys or CM-Dex:pLys:RNA in either $0.1 \mathrm{M} \mathrm{Na}^{+}$bicine buffer ( $\mathrm{pH} 8.5$ ) or $10 \mathrm{mM}$ Tris and $4 \mathrm{mM} \mathrm{MgCl}$ ( $\mathrm{mH} 8.0$ ). The chamber was then loaded onto a fluorescence microscope (Supplementary Section 1) which was focused on the cold wall and images were taken every $1-10 \mathrm{~s}$ for a certain timeframe (usually $1-2 \mathrm{~h}$ ) using custom-built software written in LabVIEW.

Imaging was performed with a custom-built fluorescence microscope equipped with a blue light-emitting diode $(470 / 29 \mathrm{~nm})$, an amber light-emitting diode $(590 / 14 \mathrm{~nm})$, excitation filters $(482 / 35 \mathrm{~nm}, 588 / 20 \mathrm{~nm})$, a dual bandpass dichroic mirror (transmission edges at 505 and $606 \mathrm{~nm}$ ), a $5 \times$ objective and an image splitter containing a longpass filter $(600 \mathrm{~nm})$ and emission filters $(536 / 40 \mathrm{~nm}$, $630 / 50 \mathrm{~nm}$ ). This filterset allowed for the imaging of FITC and ROX respectively. The crosstalk between the channels was calculated following a standard protocol ${ }^{27}$ (Supplementary Section 1). A Stingray-F145B ASG camera (ALLIED Vision Technologies Gmbh) was used to acquire images. The voltages to the light-emitting diodes and the camera were controlled with the LabVIEW software (a schematic of the microscope is shown in Supplementary Fig. 1). Image analysis of the droplets was performed using ImageJ or LabVIEW. The raw data from the two different illumination channels were merged together to generate the composite dual fluorescence image.

\section{Data availability}

The datasets generated during and/or analysed during the current study are available via the following: https://doi.org/10.17617/3.6n.

\section{Code availability}

The code for the analysis of the particle sizes and for the dual-channel images is available via Edmond (the Open Research Data Repository of the Max Planck Society) at https://doi.org/10.17617/3.6n.

\section{Acknowledgements}

Financial support came from the European Research Council (ERC Evotrap, grant no. 787356 (D.B.)), the Simons Foundation (grant no. 327125 (D.B.)), the Quantitative Biosciences Munich Graduate School (QBM), MaxSynBio Consortium (jointly funded by the Federal Ministry of Education and Research (Germany) and the Max Planck Society (T.-Y.D.T.)), the MPI-CBG, the Cluster of Excellence Physics of Life of TU Dresden (grant no. EXC-1056 (T.-Y.D.T.)) and the VW foundation- "Life" initiative (grant nos. 92857 (T.-Y.D.T.) and 94743 (C.B.M., D.B.)), Deutsche Forschungsgemeinschaft (DFG, German Research Foundation)-Project-ID 364653263-TRR 235 (CRC235), Project P08 (C.B.M.). We thank L. Keil for sharing his expertise in the preparation of the setup for imaging and for programming support and S. Janosch (MPI-CBG) for assistance with archiving and making available the source data.

\section{Author contributions}

A.I., T.-Y.D.T., C.B.M. and D.B. designed the study. A.I., T.-Y.D.T., J.S. and A.K. performed the experiments. A.I. analysed the data. A.I., T.-Y.D.T., J.S., A.K., C.B.M. and D.B. wrote the manuscript.

\section{Funding}

Open access funding provided by Max Planck Society.

\section{Competing interests}

The authors declare no competing interests.

\section{Additional information}

Supplementary information The online version contains supplementary material available at https://doi.org/10.1038/s41557-021-00830-y.

Correspondence and requests for materials should be addressed to

Dieter Braun or T.-Y. Dora Tang.

Peer review information Nature Chemistry thanks the anonymous reviewers for their contribution to the peer review of this work.

Reprints and permissions information is available at www.nature.com/reprints. 